\title{
Distributed Secondary Voltage Control for DC Microgrids with Consideration of Asynchronous Sampling
}

\author{
Guannan Lou ${ }^{1,2, *(\mathbb{D})}$, Yinqiu Hong ${ }^{1}\left(\mathbb{D}\right.$ and Shanlin $\mathrm{Li}^{1}$ \\ 1 School of Electrical Engineering, Southeast University, Nanjing 210096, China; hyq97820@163.com (Y.H.); \\ sli9808@seu.edu.cn (S.L.) \\ 2 Jiangsu Provincial Key Laboratory of Smart Grid Technology and Equipment, Southeast University, \\ Nanjing 210096, China \\ * Correspondence: bingzhi0828@163.com
}

check for

updates

Citation: Lou, G.; Hong, Y.; Li, S Distributed Secondary Voltage Control for DC Microgrids with Consideration of Asynchronous Sampling. Processes 2021, 9, 1992 https://doi.org/10.3390/ pr9111992

Received: 20 October 2021

Accepted: 31 October 2021

Published: 8 November 2021

Publisher's Note: MDPI stays neutral with regard to jurisdictional claims in published maps and institutional affiliations.

Copyright: (c) 2021 by the authors. Licensee MDPI, Basel, Switzerland. This article is an open access article distributed under the terms and conditions of the Creative Commons Attribution (CC BY) license (https:/ / creativecommons.org/licenses/by/ $4.0 /)$.

\begin{abstract}
This paper studies the distributed secondary control of DC microgrids (MGs) in the case of asynchronous sampling, including both the stability condition and accurate consensus algorithm. The asynchrony means that the update actions of each distributed generation (DG) based on the local information and information received from neighbors are independent of the actions of others at sampled discrete times, which would cause deviation from the accurate convergence and even lead to instability in the worst case. First, a small-signal model of MG installed with secondary voltage control is established to include the individual sampling periods. A stability criterion based on the periodic continuity of sampling instant offset is thus formulated to reveal a stability mapping of multiple sampling. By quantifying the accuracy deviations caused by the asynchrony, an improved ratio consensus strategy is proposed that allows the deviation to be estimated accurately via an auxiliary signal and compensated with respect to the eventual equilibrium to produce an exact solution. Our approach customizes the stability and accuracy for distributed secondary control considering asynchronous sampling in MG, which has been ignored in most existing literature. The effectiveness of the proposed methodology is verified by simulations.
\end{abstract}

Keywords: asynchronous sampling; accurate consensus; convergence deviation; microgrid; secondary control

\section{Introduction}

With the increasing penetration of renewable energy resources, the microgrid (MG) has emerged as a promising concept for integration of distributed generations (DGs), storages and loads within an identifiable electrical boundary [1-3]. Typically, an MG can operate while connected to the main grid, and the most appealing advantage of MG is its ability to island itself to form an uninterruptible power supply [4]. With increased DGs, storages and loads being used in power systems, the incumbent power generation paradigm is being developed toward a more DC future because of advantages including simple structure and high efficiency [5-7], where an appropriate hierarchical control is indispensable to guarantee reliable and efficient operation of MG systems. To realize spontaneous power sharing without critical communication links, primary control is often implemented using the droop paradigm, which would lead to poor power sharing because of the distinct output impedance and voltage deviations involved.

To overcome these disadvantages of primary control, secondary control schemes ranging from centralized to distributed approaches have been proposed. Centralized control assumes the use of a microgrid centralized controller (MGCC) and a complex communication network that reduces system reliability and increases its sensitivity to single points-of-failure [8-10]. Inspired by the multiagent cooperative technology, distributed secondary control, where each DG exchanges information with its immediate neighbors via local networks, was proposed in [11] and has attracted considerable interest in numerous 
literatures [12-17]. In ref. [12], the concept of "virtual voltage drop" was introduced to realize current sharing and voltage restoration in DC MG regardless of the type of resistance. A distributed robust control method has been proposed in [13] to implement secondary control in cases of system uncertainties. In ref. [14], a novel unified distributed control strategy was proposed to realize seamless operation mode transitions. By integrating an eventtriggered mechanism, a periodic distributed controller was presented, which allowed the information transmission to be both intermittent and relaxed [15]. In ref. [16], an additional controller for the droop coefficient was introduced to improve the system's current sharing performance. In ref. [17], through the distributed iterative learning method, the secondary control in MG was realized without accurate knowledge of the system parameters.

Generally, a control cycle consists mainly of information sampling, updating and processing, as well as command transmission and execution [18], which initiates from the information sampling stage. Most of the existing literature on distributed secondary control is based on the assumption that the sampling actions of heterogeneous DGs are triggered in the same time sequence, i.e., using synchronous sampling instants. However, each DG's update actions based on the local sampled local information and information received from neighboring units are independent of others' in the sense that each DG is allowed to adjust its dynamics intermittently and dependently, which is inherent behavior in distributed control systems. As such, completely synchronous information exchange is almost unobtainable without the aid of synchronizers, while asynchronous information exchange among DGs can definitely lead to data interleaving and even disorder $[19,20]$, which would then cause persistent convergence deviations from accurate solutions or even system instability. Additionally, the majority of DGs are integrated into an MG via power electronic inverters and because of their negligible inertia, the DGs can respond rapidly to control commands composed of distinctly sampled information; this in turn makes the cooperativity and controllability of distributed control approach more challenging. It should be noted here that the asynchrony of distributed information exchange has been demonstrated in previous research $[21,22]$ that concerned multiple transmission delays; however, these works addressed delay-dependent stability only ignoring consensus accuracy, while the impact of asynchronous sampling on the overall performance is much more sophisticated because of the accumulated time instant offset.

Motivated by the research gap mentioned above, this paper investigates distributed secondary control for MGs taking the impact of asynchronous sampling into consideration. Using a small-signal dynamic model embedded with diverse sampling periods, the sampling margin of DGs is determined. To address the convergence deviation caused by asynchrony, an improved ratio consensus algorithm is proposed based on deviation compensation. The main contributions are listed below.

(1) Given that each DG unit in a distributed control system updates its dynamics independently, the distributed secondary control in MG with regard to asynchronous sampling is first investigated in this paper, including both the stability condition and an accurate consensus algorithm.

(2) A small-signal MG model with distributed secondary control is established using the normalized periodicity of sampling offset among the interactive DGs, which allows for the stability assessment of MG with asynchronous sampling.

(3) The equilibrium deviation caused by the asynchrony of the distributed information exchange among DGs is analyzed quantitatively. Then, an accurate distributed control based on ratio consensus is proposed, where the deviation is estimated and then compensated via an auxiliary state observer timely.

The remainder of this paper is organized as follows. Section 2 reviews MG hierarchical control and formulates the asynchronous sampling concerns; a small-signal model is then proposed, with sampling stability criteria in Section 3. An improved ratio consensus algorithm is proposed in Section 4. Section 5 validates the effectiveness of proposed method by simulations; finally, conclusions are provided in Section 6 . 


\section{MG Hierarchical Control and Problem Formulation}

\subsection{Primary Control and Distributed Secondary Control}

The hierarchical control scheme is widely applied in the MG field using the control structure presented in Figure 1 [23], where each hierarchy is endowed with significant control objective and different timescale. As stated in ref. [24], the bandwidths for the voltage loop and current loop in primary control are $5 \mathrm{kHz}$ and $20 \mathrm{kHz}$, respectively, while that for secondary control is $30 \mathrm{~Hz}$.

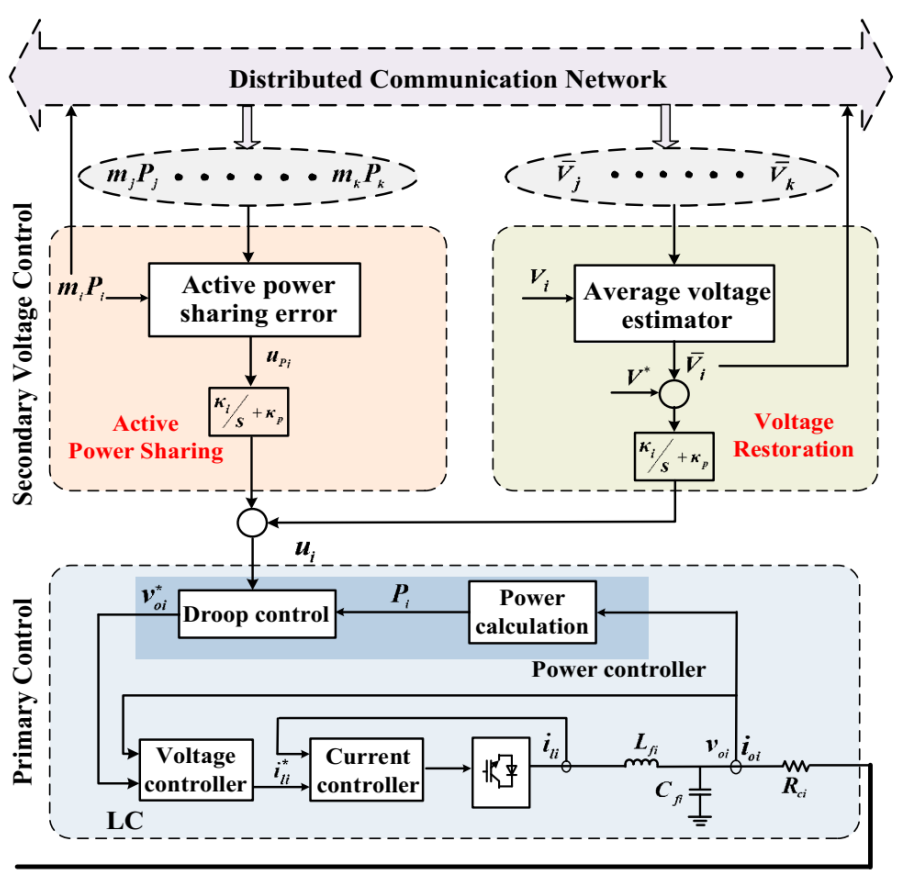

DC microgrid

Figure 1. Hierarchical control structure for DC microgrid.

Droop control is adopted as the primary control approach to ensure system stability through voltage and current regulators. The voltage loop is responsible for regulating output voltage to the set-point and a reference for the current loop is also supplied. The error between the actual and expected currents will be calculated and fed into the inner loop to determine the duty cycle to generate PWM for converters [25]. In summary, the complete droop control dynamics can be expressed as [26]:

$$
\begin{aligned}
& \frac{d \theta}{d t}=V_{n i}-m_{i} P_{i}-V_{i} \\
& i_{r e f}=k_{p v}\left(V_{n i}-m_{i} P_{i}-V_{i}\right)+k_{i v} \theta \\
& \frac{d \vartheta}{d t}=i_{r e f}-i_{i} \\
& d_{i}=k_{p i}\left(i_{r e f}-i_{i}\right)+k_{i i} \vartheta
\end{aligned}
$$

where $V_{i}$ and $V_{n i}$ denote the output and nominal voltages, respectively; $i_{i}$ and $i_{\text {ref }}$ denote the output and reference currents, respectively; $k_{p v}$ and $k_{i v}\left(k_{p i}\right.$ and $\left.k_{i i}\right)$ are the proportional and integral gains of voltage (current) controller; $d_{i}$ represents the duty cycle; $m_{i}$ is droop coefficient; and $P_{i}$ is active power.

Distributed secondary control is then introduced to realize active power sharing and voltage restoration. Typically, the active powers must be allocated appropriately in inverse 
proportion to droop coefficients, i.e., $m_{1} P_{1}=m_{2} P_{2}=\ldots=m_{n} P_{n}$ [27]. Then, a distributed control scheme that enables elimination of the active power disagreement $u_{p i}$ is given as:

$$
u_{P i}=\sum_{j=1, j \neq i}^{n} a_{i j}\left(m_{j} P_{j}-m_{i} P_{i}\right)
$$

where $a_{i j}=1$ indicates the information exchanges between the $i$ th and the $j$ th DGs; otherwise, $a_{i j}=0$. Considering the inherent tradeoff between power sharing and local voltage restoration, the average voltage across the MG is expected to converge to a rated value. An average voltage estimator is then localized in each DG based on the dynamic consensus algorithm [21] to steer its estimated voltage to the practical average voltage:

$$
\bar{V}_{i}=V_{i}+k_{i 3} \int \sum_{j=1, j \neq i}^{n} a_{i j}\left(\bar{V}_{j}-\bar{V}_{i}\right) d t
$$

where $\bar{V}_{i}$ and $\bar{V}_{j}$ denote the average voltages from the estimators of the $i$ th and $j$ th DGs, respectively.

Then, the integrated secondary control input is deduced as

$$
u_{i}=\left(k_{p 1}+\frac{k_{i 1}}{s}\right) u_{P i}+\left(k_{p 2}+\frac{k_{i 2}}{s}\right)\left(V_{n}-\bar{V}_{i}\right)
$$

where $u_{i}$ is secondary control input; $k_{p 1}, k_{i 1}, k_{p 2}$ and $k_{i 2}$ denote the proportional and integral gains, respectively. It can be observed from (2) and (3) that the average consensus and the dynamic average consensus as basis of distributed secondary control have an important effect on system performance.

\subsection{Problem Formulation of Asynchronous Samplinlg}

In the absence of centralized synchronized clock, it is difficult for each DG to update its action at exactly the same time as others; this is inherent in distributed control system. The process of asynchronous information interaction is illustrated in Figure 2, where the secondary control of DG $i$ is triggered at the sampling instant $t_{m^{1}}^{i}$ and it receives the most recently sampled information $x^{*}$ from its neighbors, which comes from their latest update timeslots, $t_{m^{2}}^{i}$ and $t_{m^{3}}^{i}$, which lag behind $t_{m^{1}}^{i}$ with a varying offset $s_{i j, m} T_{i}$ at each sampling instant. Therefore, the signal asynchrony and further convergence deviation appear.

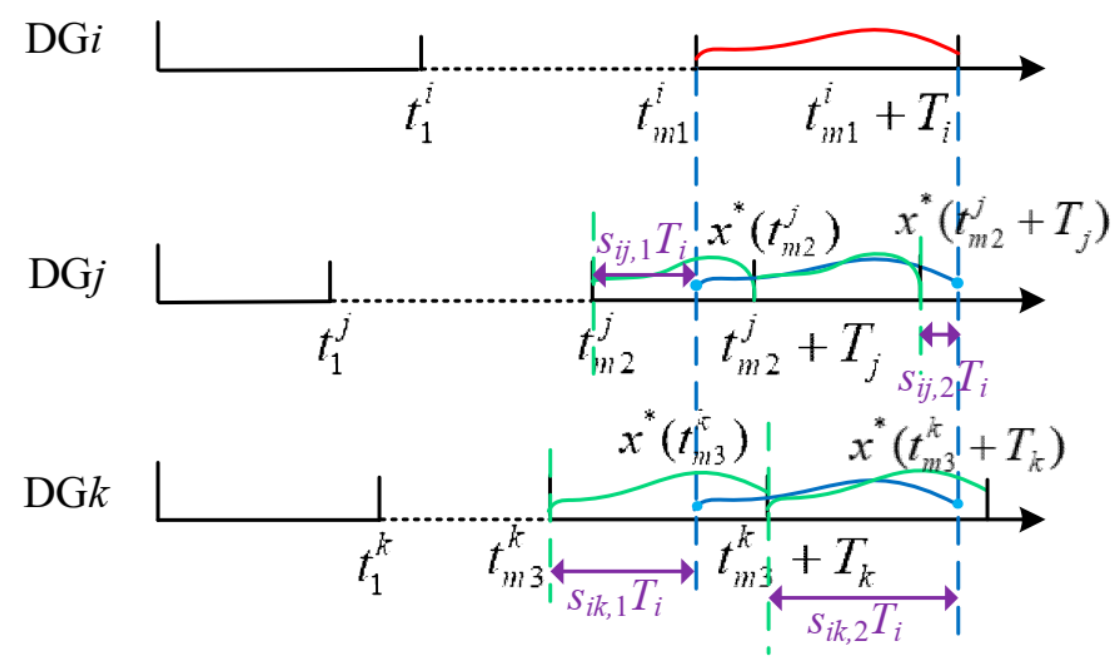

Figure 2. Schematic diagram of clock triggering process.

Suppose $p_{i j}$ is the total number of possible sampling instant offsets during information transmission from the $j$ th DG to $i$ th DG and $1 / p_{i j}$ is then denoted as the distribution 
function of sampling offset corresponding to $s_{i j, m}$ for $m \in\left[1, p_{i j}\right]$ (see the explanation in Appendix A). For more practical implementation, a discrete form of the consensus algorithm that considers asynchronous control periods is formulated as [28]:

$$
x_{i}(k+1)=\left(1-d_{i} \varepsilon T_{i}\right) x_{i}(k)+\varepsilon T_{i} \sum_{j \in N_{i}}\left[\sum_{m=1}^{p_{i j}} \frac{1}{p_{i j}} x_{j}\left(k-s_{i j, m}\right)\right]
$$

where $d_{i}$ denotes the degree of the $i$ th DG, $d_{i}=\sum_{j} a_{i j} ; \varepsilon$ represents the interaction strength and $T_{i}$ denotes the control period. Specifically, $p_{i j}$ and $s_{i j, m}$ can be formulated as:

$$
p_{i j}=\frac{\operatorname{lcm}\left(T_{i}, T_{j}\right)}{T_{i}}, s_{i j, m}=\frac{\bmod \left(m T_{i} / T_{j}\right)}{T_{i}}
$$

where $\operatorname{lcm}(x, y)$ represents the least common multiple of $x$ and $y$ and $\bmod (\cdot)$ represents its remainder.

For the integrity of time sequences for (5), the discretization period in (6) is reselected to be $s_{b} T_{i}$, where $s_{b}$ is the largest common divisor of all possible time deviations, i.e., $s_{b}=\operatorname{gcd}\left(\left\{s_{i j, m} \mid i, j \in[1, n], m \in\left[1, p_{i j}\right]\right\}\right)$, where $\operatorname{gcd}(\cdot)$ is the largest common divisor. Accordingly, (6) can then be rewritten as:

$$
x_{i}(k+h)=\left(1-d_{i} \varepsilon T_{i}\right) x_{i}(k)+\varepsilon T_{i} \sum_{j \in N_{i}}\left[\sum_{m=1}^{p_{i j}} \frac{1}{p_{i j}} x_{j}\left(k-r_{i j, m}\right)\right]
$$

where $h=1 / s_{b}$ and $r_{i j, m}=s_{i j, m} / s_{b}$.

Let $x(k+r)=\left[x_{1}(k+r), x_{2}(k+r), \ldots, x_{n}(k+r)\right]^{T}$ and $X(k)=[x(k+h-1), x(k+h-2)$, $\left.\ldots, x\left(k-r_{\max }\right)\right]^{T}$, where $r_{\max }$ represents the maximum of $r_{i j, m}$. Then, a compact form of (7) yields:

$$
X(k+h)=A_{1} X(k)
$$

where $A_{1}$ is a row stochastic matrix that is directly related to the individual sampling periods (listed in the Appendix A); 1 is an eigenvalue of $A_{1}$ along the corresponding right eigenvector $[1,1, \ldots, 1]^{T}$, which implies that all states converge to a constant value. The eigenvalues of $A_{1}$ are defined as $\lambda_{n} \leq \ldots \leq \lambda_{2} \leq \lambda_{1}$, with $u_{i}$ and $v_{i}$ as the left and right eigenvectors that correspond to $\lambda_{i}$, respectively. Therefore, the initial state $X(0)$ can be expressed using the eigenbasis of $A_{1}$ as $X(0)=\sum_{i=1}^{n}\left(u_{i} X(0)\right) v_{i}$, and then

$$
\lim _{k \rightarrow \infty} X(k)=\lim _{k \rightarrow \infty} \sum_{i=1}^{n} \lambda_{i}^{\frac{k}{h}}\left(u_{i} X(0)\right) v_{i}
$$

Considering the sufficient and necessary condition to reach consensus to be $\max _{i}$ $\geq 2\left|\lambda_{i}\right|<1$, it can be inferred that $\lim _{k \rightarrow \infty} X(\mathrm{k})=\left(u_{1} X(0)\right) v_{1}$ with $v_{1}=[1,1, \ldots, 1]^{T}$. Consequently, the final convergence value of $x$ can be expressed as:

$$
c x=u_{1} X(0)
$$

where $u_{1}$ is the left eigenvector of $A_{1}$ corresponding to the eigenvalue 1 . As shown in (10), in addition to the control parameters, the distributed consensus equilibrium is dependent on the individual sampling instants, corresponding to the sampling time offset, which is the main focus of this paper.

For the dynamic consensus, we suppose that all the dynamic states have converged to $c x^{\prime}$ at $t=t_{0}$, and the instant variation of the dynamic state at $t=t_{0}$ is then modeled as the step function $\Delta z_{i}$. The accurate average value can then be expressed as $u_{2} \Delta z$, where $u_{2}$ represents the left eigenvector of $L$ and $L=D-\Lambda$ with $D=\operatorname{diag}\left(d_{i}\right)$ and $\Lambda=\left[a_{i j}\right]$. The deviation of the consensus equilibrium from its accurate counterpart can be calculated as:

$$
\Delta c x=\left(u^{\prime}-u_{2}\right) \Delta z
$$


where $u^{\prime}=\left[\sum_{k=0}^{h} u_{1}(n k+1), \ldots, \sum_{k=0}^{h} u_{1}(n k+n)\right]^{T}$ and $\Delta z=\left[\Delta z_{1}, \Delta z_{2}, \ldots, \Delta z_{n}\right]^{T}$. Therefore, the convergence deviation of dynamic consensus is decided not only by the factor in average consensus (10) but also by the variations of the dynamic states, which makes achieving convergence accuracy more challenging.

\section{Stability Analysis in the Asynchronous Sampling Period Case}

In this section, a small-signal dynamic model of an islanded MG is established. It allows for the determination of stability region for the individual sampling periods of DGs.

\subsection{Small-Signal Modeling}

An MG model typically comprises three specific components: the inverters, the network and the loads. Given the fairly rapid response of double-loop controller when compared with that of power loop, we consider the dynamics of the power control loop only and neglect those of the double-loop [22]:

$$
V_{i}=V_{n i}-m_{i} P_{i}+u_{i}
$$

where $P_{i}$ can be measured using a low-pass filter:

$$
\dot{P}_{i}=-\omega_{f} P_{i}+\omega_{f} V_{i} I_{i}
$$

where $\omega_{f}$ denotes the filter cutoff frequency and $I_{i}$ denotes the output current of the $i$ th DG.

It is assumed that $n$ DGs are connected to the load network and the network structure is then as shown in Figure 3.

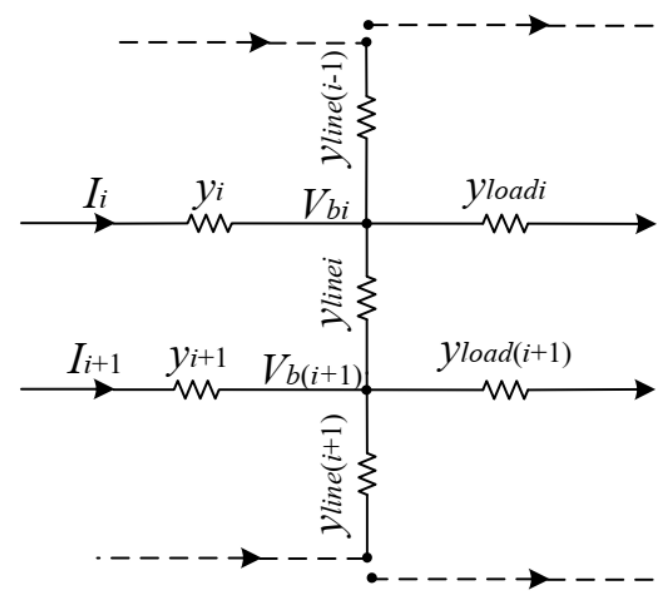

Figure 3. Network structure.

Thus, the output current can be given as:

$$
I=\Upsilon\left(V-V_{b}\right)
$$

where $I=\left[I_{1}, I_{2}, \ldots, I_{n}\right]^{T}, V=\left[V_{1}, V_{2}, \ldots, V_{n}\right]^{T}$, and $V_{b}=\left[V_{b 1}, V_{b 2}, \ldots, V_{b n}\right]^{T}$, with $V_{b i}$ denoting the voltage of the $i$ th bus; and $Y=\operatorname{diag}\left(y_{i}\right)$, where $y_{i}$ is the output admittance of the $i$ th DG.

According to the node voltage equation of the network, the $i$ th bus voltage can be determined using:

$$
y_{s i} V_{b i}=y_{\text {line }(i-1)} V_{b(i-1)}+y_{\text {linei }} V_{b(i+1)}+y_{i} V_{i}
$$

where $y_{\text {si }}=y_{\text {linei }}+y_{\text {line }(i-1)}+y_{i}+y_{\text {loadi }}$ with $y_{\text {linei }}$ and $y_{\text {loadi }}$ representing the line and load admittances, respectively. 
Combining (14) with (15), the output current and voltage can be arranged in a compact form:

$$
I=Y^{\prime} V
$$

where $Y^{\prime}=Y\left(1-Y_{S}^{-1} Y\right)$ and $Y_{S}$ is

$$
Y_{S}=\left[\begin{array}{ccccc}
y_{s 1} & -y_{\text {line } 1} & 0 & \cdots & 0 \\
-y_{\text {line } 1} & y_{s 2} & -y_{\text {line } 2} & \ddots & \vdots \\
0 & \ddots & \ddots & \ddots & 0 \\
\vdots & \ddots & -y_{\text {line }(n-1)} & y_{s(n-1)} & -y_{\text {linen }} \\
0 & \cdots & 0 & -y_{\text {linen }} & y_{s n}
\end{array}\right]
$$

Using (3), (4), (12), (13) and (16), we can then obtain

$$
\begin{gathered}
\Delta \dot{V}_{i}=-m_{i} \Delta \dot{P}_{i}+\Delta \dot{u}_{i} \\
\Delta \dot{P}_{i}=-\omega_{f} \Delta P_{i}+\omega_{f}\left(I_{i} \Delta V_{i}+\sum_{j=1}^{n} V_{i} y_{i j}^{\prime} \Delta V_{j}\right) \\
\Delta \dot{u}_{i}=k_{p 1} \sum_{j=1, j \neq i}^{n} a_{i j}\left(m_{j} \Delta \dot{P}_{j}-m_{i} \Delta \dot{P}_{i}\right)+k_{i 1} \sum_{j=1, j \neq i}^{n} a_{i j}\left(m_{j} \Delta P_{j}-m_{i} \Delta P_{i}\right)-k_{p 2} \Delta \dot{\bar{V}}_{i}-k_{i 2} \Delta \bar{V}_{i} \\
\Delta \dot{\bar{V}}_{i}=\Delta \dot{V}_{i}+k_{i 3} \sum_{j=1, j \neq i}^{n} a_{i j}\left(\bar{V}_{j}-\bar{V}_{i}\right)
\end{gathered}
$$

By consolidating (17)-(20), the complete small-signal model of system can then be deduced to be:

$$
\Delta \dot{x}=A_{2} \Delta x
$$

where $\Delta x=\left[\Delta P_{1}, \ldots, \Delta P_{n}, \Delta V_{1}, \ldots, \Delta V_{n}, \Delta \bar{V}_{1}, \ldots, \Delta \bar{V}_{n},\right]^{T}$ and $A_{2}$ is the coefficient matrix listed in the Appendix A.

Consideration of the significant distinction between the control periods of the primary and secondary layers shows that they would be implemented at their discrete times separately, and the primary control periods of DGs are supposed to be the same. The incremental forms of the droop control and power measurement equations can then be expressed as:

$$
\begin{aligned}
& \Delta V_{i}(n+1)-\Delta V_{i}(n)=m_{i} \Delta P_{i}(n)-m_{i} \Delta P_{i}(n+1)+\Delta u_{i}(n+1)-\Delta u_{i}(n) \\
& \Delta P_{i}(n+1)-\Delta P_{i}(n)=-\omega_{f} T_{s} \Delta P_{i}(n)+\omega_{f} T_{s}\left[I_{i} \Delta V_{i}(n)+\sum_{j=1}^{n} V_{i} y_{i j}^{\prime} \Delta V_{j}(n)\right]
\end{aligned}
$$

where $T_{s}$ denotes the primary control period.

Analogously, the incremental model for secondary control with consideration of the asynchrony can be formulated as:

$$
\begin{gathered}
\Delta u_{i}(n+1)-\Delta u_{i}(n)=k_{p 1} \sum_{j=1, j \neq i}^{n} a_{i j}\left[m_{j}\left(\Delta P_{j}(n+1)-\Delta P_{j}(n)\right)-m_{i}\left(\Delta P_{i}(n+1)-\Delta P_{i}(n)\right)\right] \\
+k_{i 1} T_{i} \sum_{j=1, j \neq i}^{n} a_{i j}\left[m_{j} \Delta P_{j}(n)-m_{i} \Delta P_{i}(n)\right]+k_{p 2}\left[\Delta \bar{V}_{i}(n)-\Delta \bar{V}_{i}(n+1)\right]-k_{i 2} T_{i} \Delta \bar{V}_{i}(n) \\
\Delta \bar{V}_{i}(n+1)-\Delta \bar{V}_{i}(n)=\Delta V_{i}(n+1)-\Delta V_{i}(n)+k_{i 3} T_{i} \sum_{j=1, j \neq i}^{n} a_{i j}\left[\Delta \bar{V}_{j}(n)-\Delta \bar{V}_{i}(n)\right]
\end{gathered}
$$




\subsection{Asynchronous Sampling-Dependent Stability Analysis}

As demonstrated in Figure 4, supposing that the initial instant of all DGs is uniform at $t=t_{0}$ and letting $\mathrm{T}$ be the least common multiple of all the DG control periods, i.e., $T=\operatorname{lcm}\left(T_{1}, T_{2}, \ldots, T_{n}\right)$, the staggered information interaction process continues except for the instant set $t=t_{0}+k T(k=1,2, \ldots)$ when the sampling information in MG is synchronous because of the global timeslot. Therefore, we perform the modeling and analysis within $T$, which is regarded as a normalized cycle period.

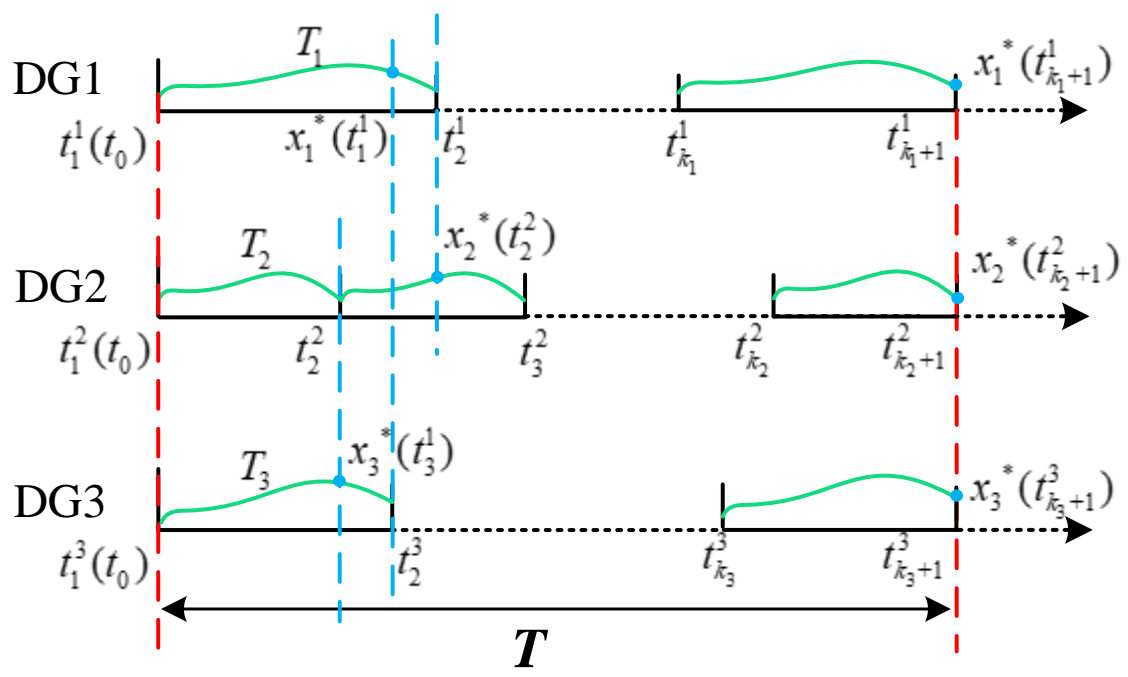

Figure 4. Periodicity in the sampling time offset during information interaction.

As stated previously, the primary and secondary control layers are operated with different control periods. The primary control instants of individual DGs within the cycle period $T$ are represented by $t_{1_{k}}, t_{2_{k}}, \ldots, t_{k}^{n}$, where $n=T / T_{s}$, and the update process of the measured powers and voltages at the primary control instant $t_{k}^{l}$ can then be expressed based on (22) and (23):

$$
\Delta \bar{x}\left(t_{k}^{l+1}\right)=W_{1} \Delta \bar{x}\left(t_{k}^{l}\right)+H_{1}\left[\Delta u\left(t_{k}^{l+1}\right)-\Delta u\left(t_{k}^{l}\right)\right]
$$

where $\Delta \bar{x}=\left[\Delta P_{1}, \Delta P_{2}, \ldots, \Delta P_{n}, \Delta V_{1}, \Delta V_{2}, \ldots, \Delta V_{n}\right]^{T} ; W_{1}$ and $H_{1}$ are the coefficient matrices presented in the Appendix A.

Here, we consider two cases: (1) if secondary control is not activated at $t=t_{l+1_{k}}$, then $\Delta u\left(t_{l+1_{k}}\right)-\Delta u\left(t_{1_{k}}\right)=0$; (2) if secondary control is triggered at $t=t_{l+1_{k}}$, then the related variables of the $i$ th DG can be updated as:

$$
\begin{gathered}
\Delta u_{i}\left(t_{k}^{l+1}\right)-\Delta u_{i}\left(t_{k}^{l}\right)=W_{i 2} \Delta \bar{x}\left(t_{k}^{l+1}\right)+W_{i 3} \Delta \bar{x}\left(\widetilde{t}_{k}\right)+G_{i 1} \Delta \bar{V}\left(t_{k}^{l+1}\right)+G_{i 2} \Delta \bar{V}\left(\widetilde{t}_{k}\right) \\
\Delta \bar{V}_{i}\left(t_{k}^{l+1}\right)=W_{i 4}\left[\Delta \bar{x}\left(t_{k}^{l+1}\right)-\Delta \bar{x}\left(\widetilde{t}_{k}\right)\right]+G_{i 3} \Delta \bar{V}_{i}\left(\widetilde{t}_{k}\right)
\end{gathered}
$$

where $x\left(\widetilde{t}_{k}\right)$ represents the state from the last secondary control instant that was recorded; $W_{i 2}, W_{i 3}, W_{i 4}, G_{i 1}, G_{i 2}$, and $G_{i 3}$ are the coefficient matrices presented in the Appendix A.

By performing $n$ iterations based on the process described above, the discrete smallsignal model of MG system within the cycle period $T$ can be arranged as:

$$
\Delta x\left(t_{k}^{l+n}\right)=D_{1} \Delta x\left(t_{k}^{l}\right)
$$

where $D_{1}$ is the coefficient matrix shown in the Appendix A.

The eigenvalues of $D_{1}$ are denoted by $\lambda_{n} \leq \ldots \leq \lambda_{2} \leq \lambda_{1}$, and then the asynchronous MG system is stable if and only if all the characteristic roots lie within the unit circle, i.e., $\max \left|\lambda_{i}\right| \leq 1$. 


\section{Improved Ratio Consensus Algorithm}

As shown in Section 2, the average consensus and dynamic consensus are two important aspects of MG secondary control, where the former is a special case of the latter based on the supposition that the local dynamic state disappears. Therefore, for the convenience of analysis, the accuracy improvement for dynamic average consensus is formulated later, and the average consensus can be dealt with in the similar way.

\subsection{Ratio Consensus Algorithm}

To address the exact observed average equilibrium of distributed control in the presence of communication delays, the ratio consensus algorithm was proposed in [29] as follows:

$$
\begin{gathered}
\dot{x}_{i}(t)=\dot{z}_{i}(t)+\varepsilon \sum_{j \neq i} a_{i j}\left[x_{j}(t-\tau)-x_{i}(t)\right] \\
\dot{y}_{i}(t)=\varepsilon \sum_{j \neq i} a_{i j}\left[y_{j}(t-\tau)-y_{i}(t)\right] \\
p_{i}(t)=\frac{x_{i}(t)}{y_{i}(t)}
\end{gathered}
$$

where $z_{i}$ is the actual dynamic state of $i$ th agent, whose average is being tracked; $x_{i}$ and $x_{j}$ are the average values from the $i$ th and $j$ th observers; $y_{i}$ and $p_{i}$ are state variable and local average observation, respectively and $\tau$ is the transmission delay.

The ratio consensus algorithm consists of two parts (30) and (31), of which the simultaneous convergence indicate the consensus of entire protocol. As stated in ref. [30], the observer of the scheme can accurately track the average dynamic states (i.e., $\left.x_{i}=\sum_{N i=1} z_{i} / N\right)$ only when $\bar{\tau}$ remains unchanged, otherwise it would lead to convergence error from the exact solution. In contrast to the unchanged $\bar{\tau}$, the asynchronous sampling case considered in this paper is notoriously complex because the probability distribution of timeslot for information exchange is time-varying during the sampling procedure, which poses challenge to the ratio consensus algorithm in accurate average.

\subsection{Improved Consensus Algorithm}

In this section, an improved algorithm is proposed to track the accurate consensus equilibrium of MG system in case of asynchronous sampling of DGs. The discrete form of this distributed average estimator is formulated as follows:

$$
\begin{gathered}
x_{i}(n+1)=z_{i}(n+1)+\varepsilon T_{i} \sum_{r=1}^{n} \sum_{j \neq i} a_{i j}\left[x_{j}\left(r-s_{i j}\right)-x_{i}(r)\right] \\
y_{i}(n+1)=g_{i}(n+1)+\varepsilon T_{i} \sum_{r=1}^{n} \sum_{j \neq i} a_{i j}\left[y_{j}\left(r-s_{i j}\right)-y_{i}(r)\right] \\
p(n+1)=p(n)+\frac{x(n+1)-x(n)}{\Delta c_{y}(n)}
\end{gathered}
$$

where $x_{i}$ and $y_{i}$ represent the estimated average values of $\mathbf{z}=\left[z_{1}, z_{2}, \ldots, z_{n}\right]$ and $g=\left[g_{1}, g_{2}\right.$, $\left.\ldots, g_{n}\right]$, respectively; $s_{i j}$ denotes the sampling time offset between the $i$ th DG and $j$ th DG, which is time-varying; $g_{i}$ is a predefined slope signal localized in each DG:

$$
\begin{gathered}
g_{i}=f_{\text {square }}(t) \cdot \min \left(k \cdot\left[\frac{2 t}{T_{g}}\right], 1\right)+\frac{3}{2}-\frac{1}{2} f_{\text {square }}(t) \\
f_{\text {square }}(t)=\frac{4}{\pi} \sum_{m=1}^{\infty} \sin \left((2 m-1) \frac{t}{T_{g}}\right) /(2 m-1)
\end{gathered}
$$


where $f_{\text {square }}(t)$ is a square function with variation range $[-1,1] ; k$ is the slope coefficient corresponding to variation dynamics of $\mathbf{z} ;[\cdot]$ represents the rounding function; $T_{g}$ is the designed period of $g_{i}$. It can be observed from (36) and (37) that $g_{i}$ varies with $k$ every $T_{g}$ and $\left|g_{\max }-g_{\min }\right|=1 . \Delta c_{y}=\left|c_{y}-c_{y}{ }^{\prime}\right|$, with $c_{y}$ and $c_{y}{ }^{\prime}$ are the estimated average of $g_{i}$ with respect to $g_{\max }$ and $g_{\text {min }}$, respectively.

The control diagram of the proposed consensus scheme consists of three parts, the traditional dynamic consensus, deviation estimation and compensation as presented in Figure 5. Because of asynchronous information exchange, $x_{i}$ deviates from the accurate average value using traditional observer and the relevant deviation depends on the distribution of sampling instant offset based on Section 2, which is difficult to obtain. It is obvious that $z_{i}$ and $g_{i}$ in each DG can be updated using the uniform control period; hence they share the same time offset distribution. Similar to the time delay case in ref. [27], we denote the deviation factor (DF) here as the ratio of estimated average variation of observer and actual average variation to indicate the impact of asynchronous sampling on consensus accuracy. For the definition of (36), it is easy to calculate DF of $g_{i}$ as

$$
D F=\frac{1}{1+\varepsilon \overline{\mathcal{S}}}=\frac{\left|\Delta c_{y}\right|}{|\Delta g|}=\left|\Delta c_{y}\right|
$$

where $\bar{s}$ represents the mean of the sampling time offset; $\Delta g$ is the actual average variation, $|\Delta g|=\left|g_{\max }-g_{\min }\right|=1$ here. $\Delta c_{y}$ updated every time when $g_{i}$ reaches its maximum or minimum value and is observed locally so DF is accessible for each DG.

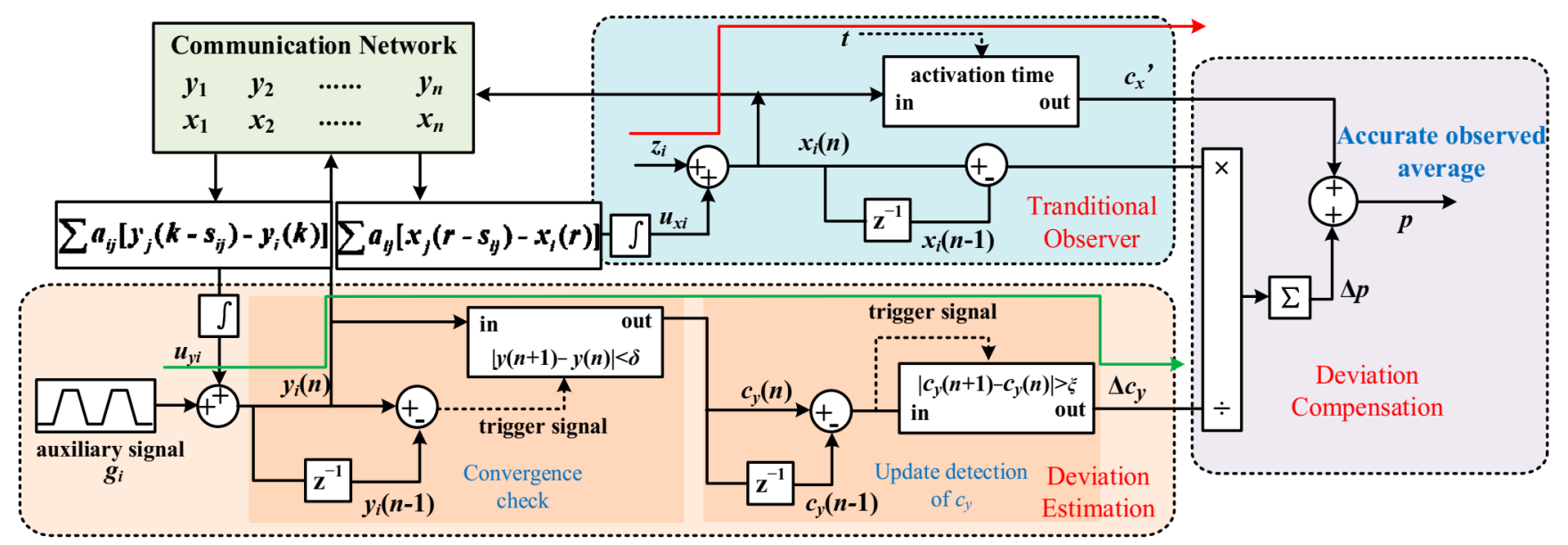

Figure 5. Control diagram for the proposed dynamic consensus algorithm.

Alternatively, the observed average variation of dynamic state $z_{i}$ can be expressed as:

$$
x(n+1)-x(n)=\frac{1}{1+\varepsilon \bar{s}} u_{2} \Delta z
$$

where $u_{2} \Delta z$ denotes the actual variation of average states.

Considering that $z_{i}$ under track holds the same sampling time offset $\bar{s}$ as $g_{i}$, they also have the same DF. Therefore, the actual variation can thus be obtained without the measurement of time offset even in face of a varying sampling offset during state exchange. Based on (38) and (39), it yields,

$$
u_{2} \Delta z=\frac{x(n+1)-x(n)}{\Delta c_{y}}
$$

Then, the accurate average states can be observed using

$$
u_{2} z=c_{x}{ }^{\prime}+\sum u_{2} \Delta z
$$


where $c_{x}{ }^{\prime}$ is the consensus value obtained from the traditional dynamic observer as shown in Figure 5. Combining (40) and (41), the improved dynamic observer can be formulated in (35) with the deviation estimated and compensated step by step, to arrive at an exact consensus solution eventually.

As discussed above, the deviation estimation is of most significance for the proposed accurate consensus algorithm. To guarantee properly determination of $\Delta c_{y}$ every step, a convergence check and an update detection module are applied as shown in Figure 5. The judgment condition for the convergence check can be expressed as $|y(n+1)-y(n)|$ $<\delta$, where $\delta$ is a preset upper error bound. When the condition above is satisfied, it can be deduced that $y$ converges to a new equilibrium, which then become the updated output; otherwise, the previous value will hold, so $c_{y}$ is available if a step variation in $g_{i}$. Additionally, $\left|c_{y}(n+1)-c_{y}(n)\right|$ is measured to detect the update action of $c_{y}$, where $\left|c_{y}(n+1)-c_{y}(n)\right|>\xi$ indicates that $c_{y}$ has updated to a new value with a threshold $\xi$ and $\Delta c_{y}$ can then be obtained as $\left|c_{y}(n+1)-c_{y}(n)\right|$. In order to avoid the misjudgment of $\Delta c_{y}$ update, it should be satisfied that $\xi>\delta$. Ultimately, a precise average estimation can be realized due to the accessible compensation. As average consensus is a special case of dynamic consensus, the proposed algorithm can also be utilized for accurate average convergence, which is demonstrated from power sharing.

\section{Simulation Results}

Case studies are performed to investigate the secondary control performance under asynchronous sampling conditions using a test DC microgrid shown in Figure 6, where the system and control parameters are listed in Table 1 and the desired power sharing ratio among the three DG units is 1:1:1. For simplification, the test system consisting of three DGs is considered, whereas the method proposed in this paper is also applicable to systems with larger scales. Moreover, note that this paper focuses on the effect of distinct sampling, so a cycle network is adopted which can be modified to other connected forms. Simulation results using MATLAB/Simulink are utilized to verify the effectiveness of the proposed criterion.

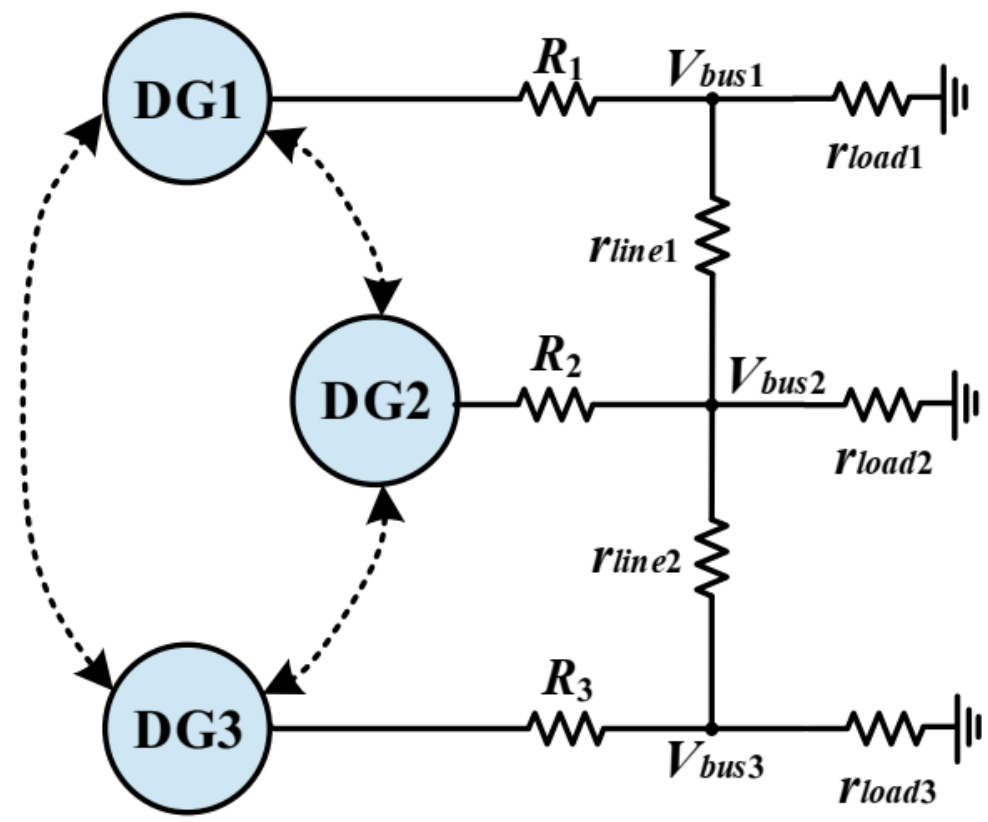

Figure 6. Structure of the test DC microgrid. 
Table 1. Network and control parameters.

\begin{tabular}{cccc}
\hline Parameter & Value & Parameter & Value \\
\hline MG voltage & $800 \mathrm{~V}$ & \multicolumn{2}{c}{ Connection impedances } \\
\hline DG power ratings & \multicolumn{2}{c}{$R_{1} / R_{2} / R_{3}$} & $0.15 \Omega / 0.3 \Omega / 0.4 \Omega$ \\
\hline$D G 1, D G 2, D G 3$ & \multicolumn{2}{c}{ Line impedances } \\
\hline Voltage droop coefficient & \multicolumn{2}{c}{$0.2 \Omega$} \\
\hline$m_{P 1}, m_{P 2}, m_{P 3}$ & $1 \times 10^{-3} \mathrm{~V} / \mathrm{W}$ & \multicolumn{2}{c}{ Load ratings } \\
\hline Control parameters & \multicolumn{2}{c}{$r_{\text {load } 1}$} \\
\hline$k_{i 1} / k_{p 1}$ & \multicolumn{2}{c}{$r_{\text {load } 2}$} \\
\hline$k_{i 2} / k_{p 2}$ & $6 / 0.5$ & $60 \Omega / 5 \Omega$ \\
\hline$k_{i 3}$ & $10 / 0.5$ & $r_{\text {load } 3}$ \\
\hline
\end{tabular}

\subsection{Stability Analysis}

(1) Theoretical stability region of control periods: The control period of each DG is assumed to be independent of others and the sampling-dependent stability region is calculated using the method proposed in Section 3. Figure 7a-c shows the dominant root loci of state matrix $D_{1}$ in (29), revealing the critical sampling periods which occupies the unit cycle. It can be found that generally as the control period increases, the eigenvalues move from the inside of unit circle to the outside, which indicates a less stable system. Another significant phenomenon is that the system could also become unstable with a great disagreement among the control periods. It can be observed that when $T_{1}=0.12 \mathrm{~s}$ and $T_{2}=0.16 \mathrm{~s}$, the MG system becomes unstable if $T_{3} \leq 0.04 \mathrm{~s}$ due to the notable sampling inconsistence. This is in contrast to the widely held opinion that the smaller control period is, the more stable system is and is crucial for the design of control period. Similarly, the stability mapping with regard to multiple independent control periods are yielded and the results are depicted in Figure $7 \mathrm{~d}$.

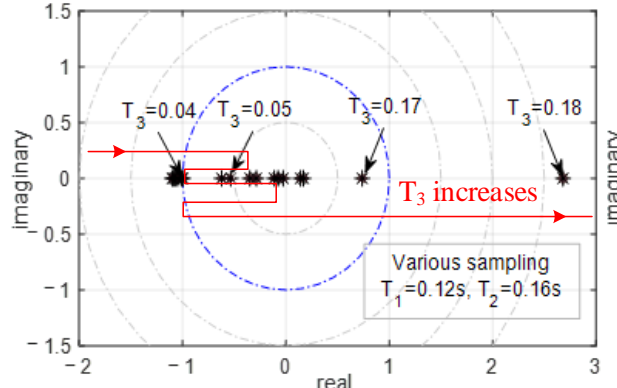

(a)

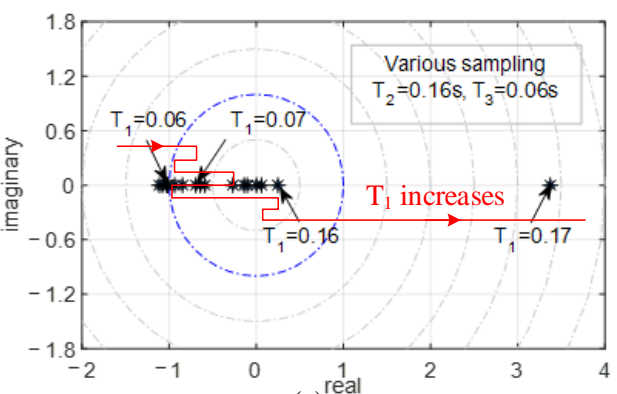

(c)

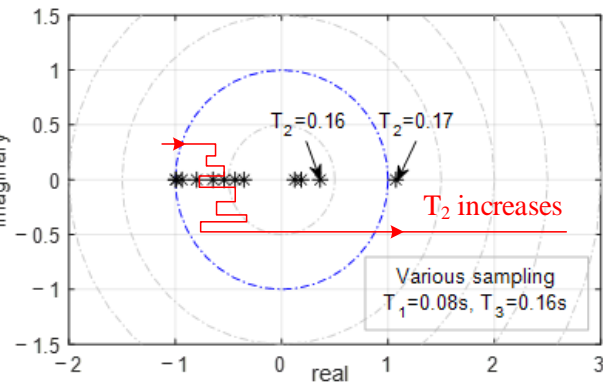

(b)

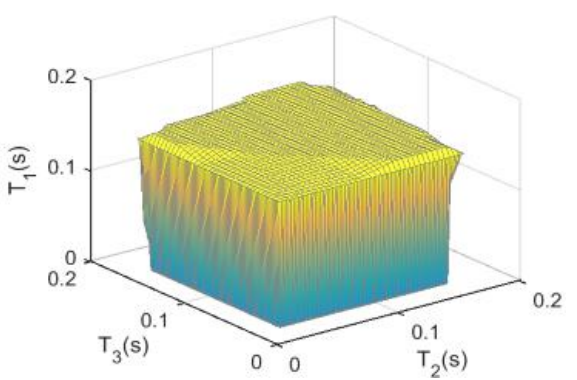

(d)

Figure 7. (a) Evaluation of $T_{3}$ when $T_{1}$ and $T_{2}$ are fixed. (b) Evaluation of $T_{2}$ when $T_{1}$ and $T_{3}$ are fixed. (c) Evaluation of $T_{1}$ when $T_{2}$ and $T_{3}$ are fixed. (d) Stability region of sampling periods for $T_{1}$, $T_{2}$ and $T_{3}$. 
(2) Simulation verification of stability region: To validate the theoretically calculated stability region, simulation studies are performed with $T_{1}=0.12 \mathrm{~s}, T_{2}=0.16 \mathrm{~s}$ and different $T_{3}$. The MG is initially controlled by droop control and secondary voltage control is activated at $t=3 \mathrm{~s}$. The effectiveness of secondary control is verified with the consistent control period $T_{1}=T_{2}=T_{3}=0.1 \mathrm{~s}$ as shown in Figure 8a, where the voltage deviates and proper power sharing fails initially and after launching secondary control, the voltages restore and power is accurately apportioned. Figure $8 \mathrm{~b}-\mathrm{d}$ display the corresponding responses for asynchronous cases. For $T_{3}=0.07 \mathrm{~s}$, the simulation results in Figure $8 \mathrm{~b}$ experience violent but decaying oscillations. With an increased control period $T_{3}=0.18 \mathrm{~s}$, the MG becomes unstable due to the growing oscillations. Then, the $T_{3}$ stability region lies within $[0.07 \mathrm{~s}, 0.18 \mathrm{~s}]$ for $T_{1}=0.12 \mathrm{~s}$ and $T_{2}=0.16 \mathrm{~s}$, which coincides with the theoretically calculated range [0.05 s, $0.017 \mathrm{~s}$ ] (see Figure 7a). However, for a relatively small value $T_{3}=0.04 \mathrm{~s}$, the system tends to be unstable as shown in Figure $8 \mathrm{~d}$ because of the notable sampling disagreement. Therefore, we confirm the validity of the proposed stability analysis criterion.

\subsection{Accuracy Analysis}

(1) Theoretical Analysis of Consensus Deviation. Case studies are developed with the sampling set $\left(T_{1}=0.07 \mathrm{~s}, T_{2}=0.08 \mathrm{~s}\right.$ and $\left.T_{3}=0.05 \mathrm{~s}\right)$ to verify the accurate consensus convergence of the proposed algorithm. In this case, the average sampling time offset $\bar{s}_{i j}$ during information interactions between any two DGs can be calculated to be $\bar{s}_{12}=0.035 \mathrm{~s}$, $\bar{s}_{13}=0.02 \mathrm{~s}, \bar{s}_{21}=0.03 \mathrm{~s}, \bar{s}_{23}=0.02 \mathrm{~s}, \bar{s}_{31}=0.03 \mathrm{~s}$, and $\bar{s}_{32}=0.035 \mathrm{~s}$ in this case. Provided that the threshold of average sampling time offset estimation is $1 / 5 \bar{s}_{\min }$, it can be derived that $N_{12} T_{1}=0.484, N_{13} T_{1}=0.245, N_{21} T_{2}=0.46, N_{23} T_{2}=0.28, N_{31} T_{3}=0.2875$, and $N_{32} T_{3}=0.34$.

(2) Simulation verification for accurate consensus. To illustrate the superiority of proposed consensus algorithm, a comparison with conventional consensus scheme is performed with the same operation. Initially, the system operates under the individual effect of droop control, where the average voltage is remarkably lower than the nominal value and the active power sharing is inappropriate among DGs. After $t=4 \mathrm{~s}$, the secondary control launches and the states of the system tend to adjust to the ideal operating equilibrium. In the traditional dynamic consensus protocol as shown in Figure 9, when the observed average voltage value is regulated to the reference $800 \mathrm{~V}$, an approximate deviation of $13.5 \mathrm{~V}$ appears in the estimated average voltage because of the asynchronous sampled information among DGs. Further, we can observe the voltage variation on the occasion of activation of secondary control as $\Delta z=[81 \mathrm{~V}, 90 \mathrm{~V}, 102 \mathrm{~V}]^{T}$ via simulation responses, and then the theoretical equilibrium deviation is calculated to be $\Delta c x=19.16 \mathrm{~V}$ using the method proposed in Section 2, which is roughly identical to the actual value $13.5 \mathrm{~V}$. As shown in Figure 10, the observed average voltage of proposed consensus algorithm can exactly track the actual average voltage due to the real-time deviation estimation and compensation so that the accurate secondary control is maintained. Moreover, the total output power of the conventional method in Figure 9a is nearly $10 \mathrm{~kW}$ higher than that in Figure 10a because of the convergence error. Therefore, the accuracy and effectiveness of the proposed distributed secondary control is verified despite asynchronous sampling instants among individual DGs. 

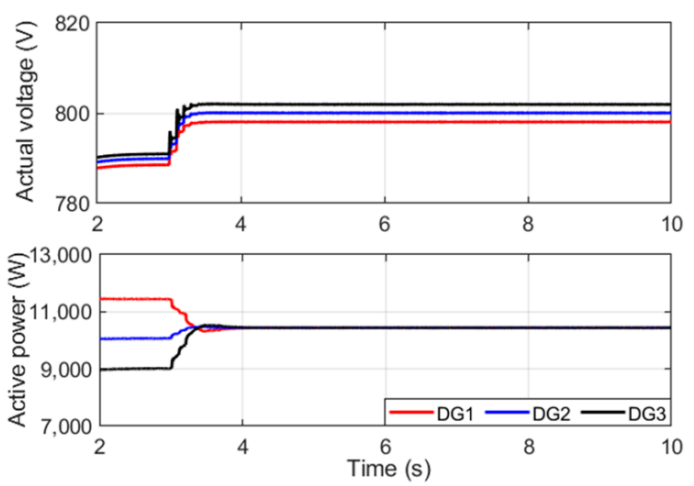

(a)
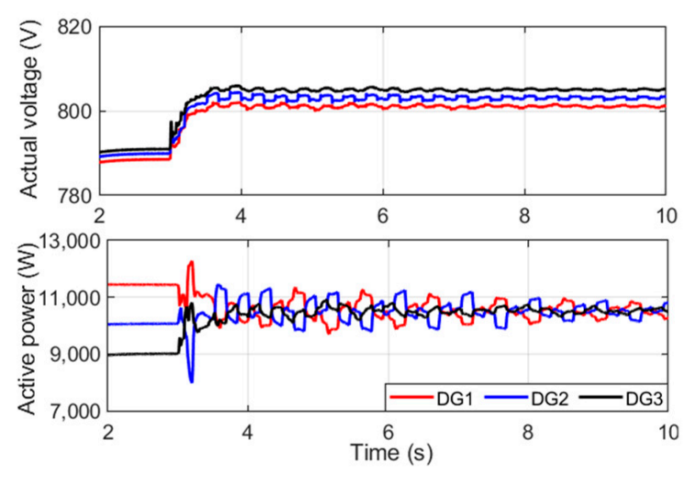

(b)
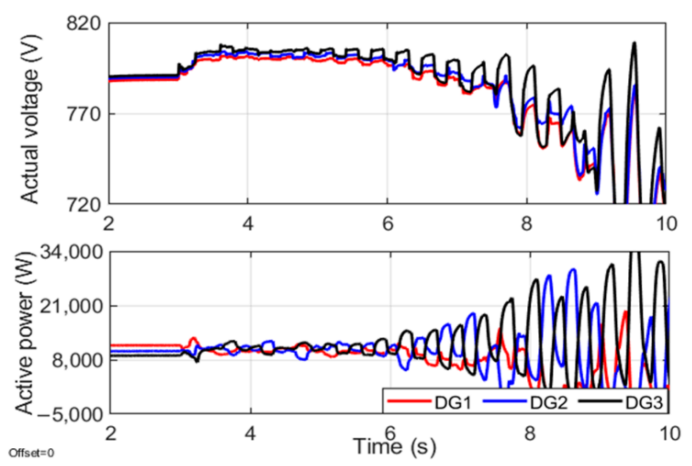

(c)
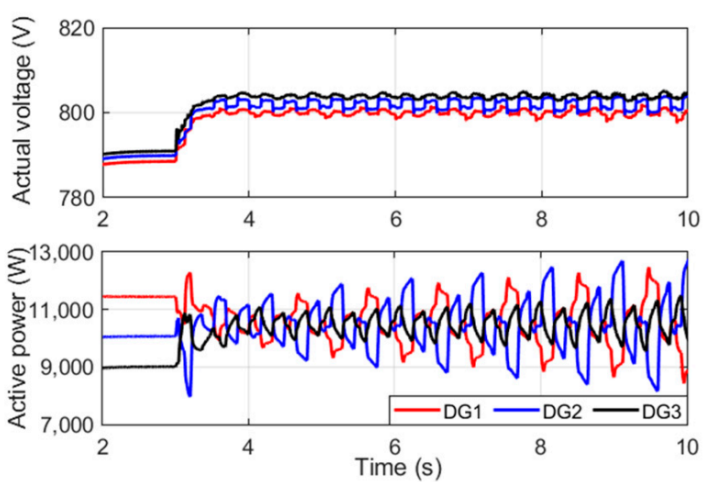

(d)

Figure 8. Simulations with different control periods. (a) $T_{1}, T_{2}, T_{3}=0.1 \mathrm{~s}$. (b) $T_{1}=0.12 \mathrm{~s}, T_{2}=0.16 \mathrm{~s}$, $T_{3}=0.07 \mathrm{~s}$. (c) $T_{1}=0.12 \mathrm{~s}, T_{2}=0.16 \mathrm{~s}, T_{3}=0.18 \mathrm{~s}$. (d) $T_{1}=0.12 \mathrm{~s}, T_{2}=0.16 \mathrm{~s}, T_{3}=0.04 \mathrm{~s}$. 


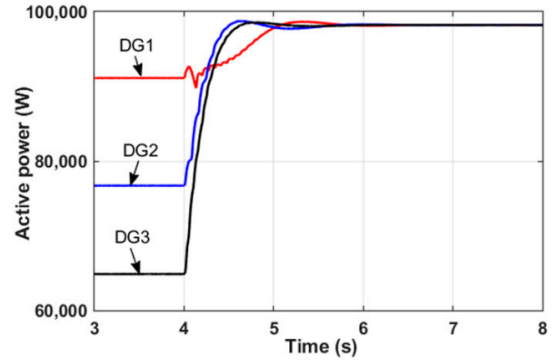

(a)

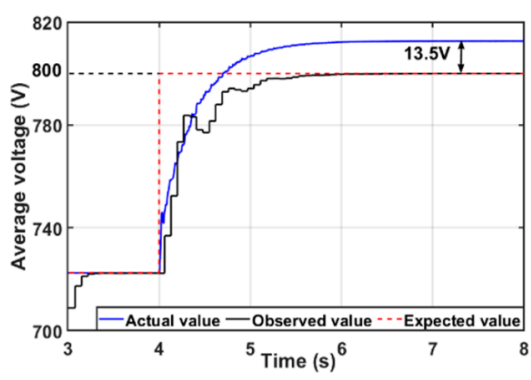

(b)

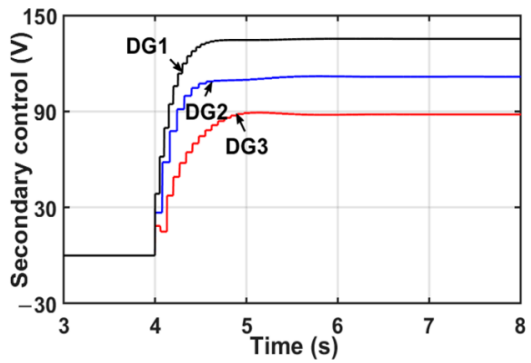

(c)

Figure 9. Simulation results for asynchronous sampled system with conventional algorithm. (a) Active powers. (b) Average voltage. (c) Secondary control inputs.

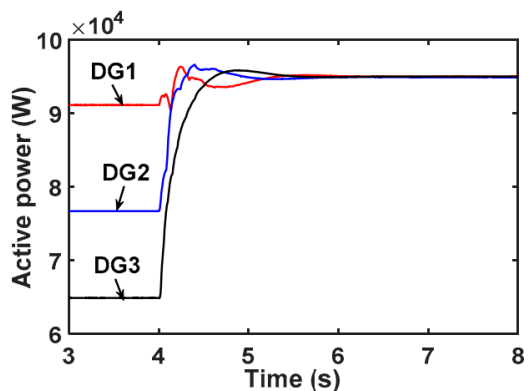

(a)

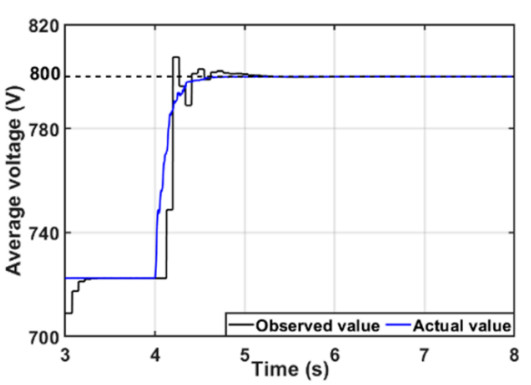

(b)

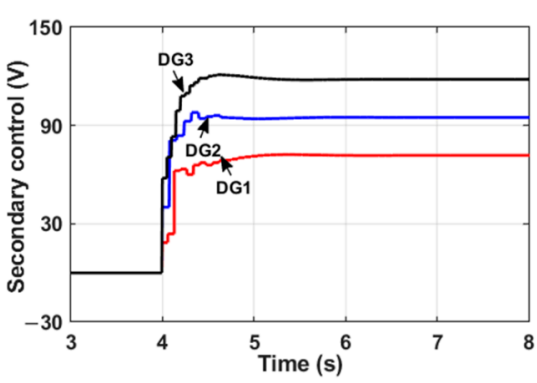

(c)

Figure 10. Simulation results for asynchronous sampled system with proposed algorithm. (a) Active powers. (b) Average voltage. (c) Secondary control inputs.

To further demonstrate the effectiveness of the proposed strategy in different cases, the cases of load variation and topology switch are developed, with their simulation results displayed in Figures 11 and 12 respectively. In the first case, an additional load $(30 \Omega)$ is connected to the voltage bus $V_{\text {bus } 2}$ at $t=6 \mathrm{~s}$. As can be visualized from Figure 11, the active power of each DG increases and the average voltage decreases at $t=6 \mathrm{~s}$, whereas the average voltage can still restore to the nominal value accurately and the active power would be stable at a new operation equilibrium after a transient process with the assistance of the proposed control strategy.

Further, the topology switch is considered in the second case where the communication link between DG1 and DG3 in the topology is set to be disconnected at $t=4.5 \mathrm{~s}$. As is shown in Figure 12, the convergence rate would become slow due to the reduction in the number of communication links, whereas accurate power sharing and voltage regulation can still be achieved ultimately using the proposed strategy. 

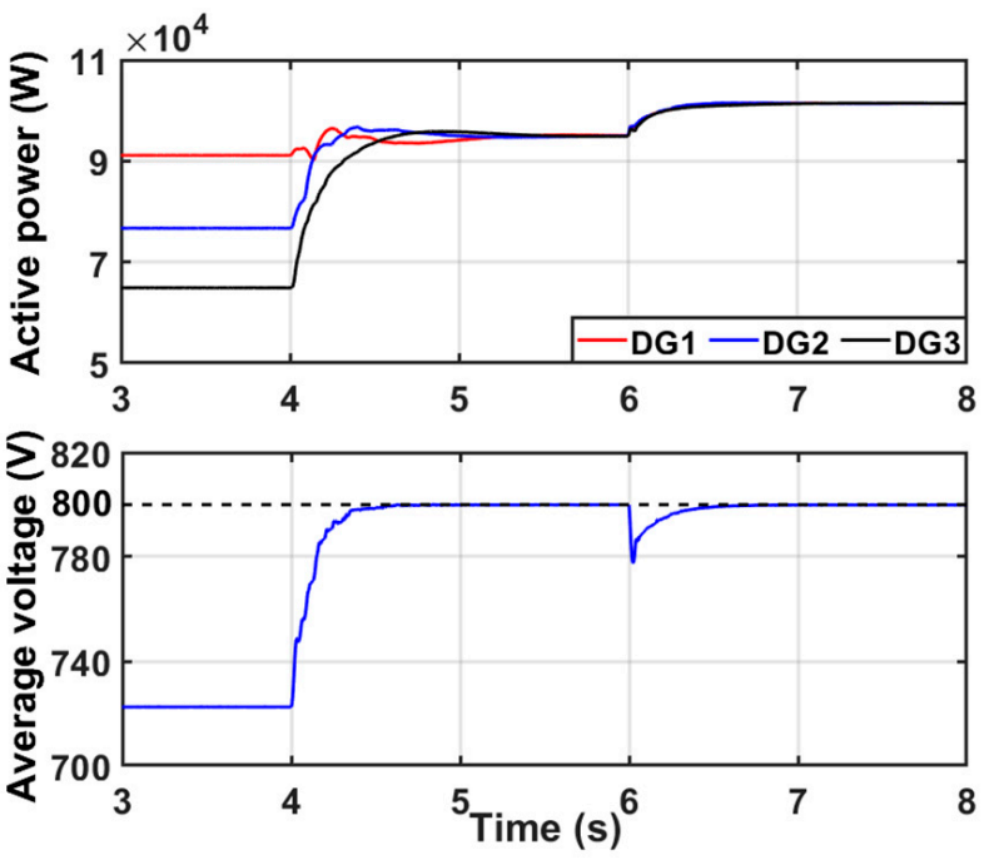

Figure 11. Simulation results in case of load variation under proposed algorithm.
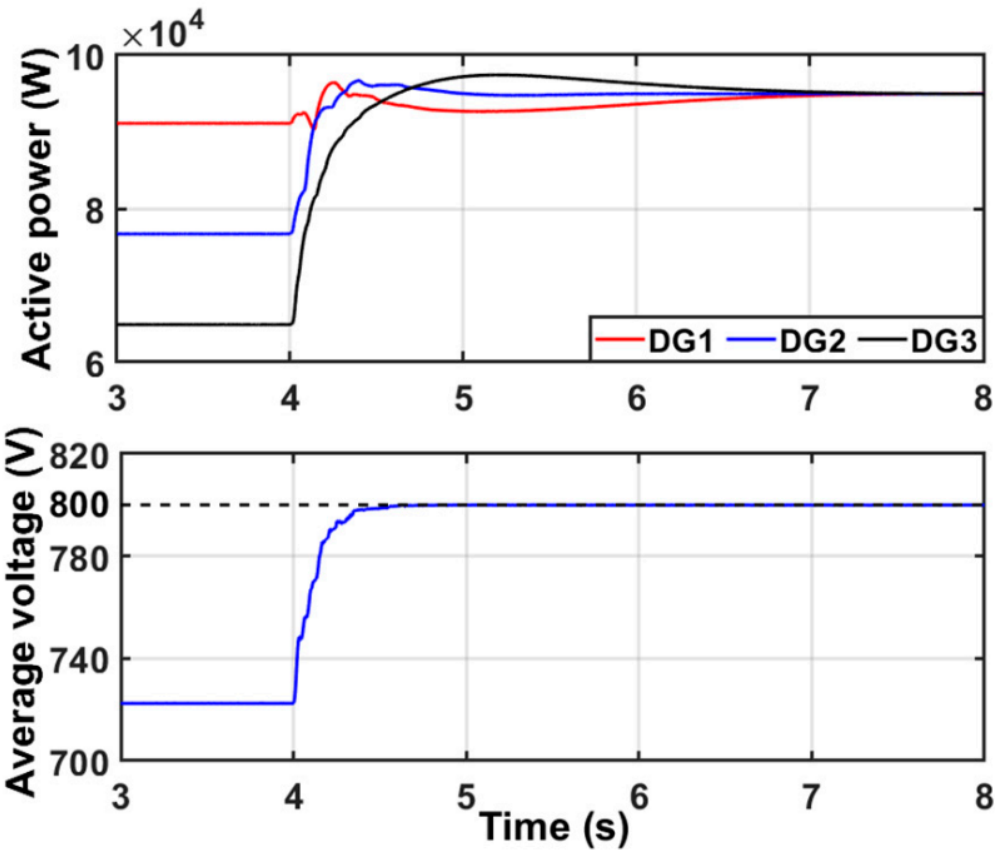

Figure 12. Simulation results in case of topology switch under proposed algorithm.

\subsection{Discussion of the results}

According to the simulation results, it can be deduced that:

(1) The simulation results accord well with the theoretical analysis, which verifies the effectiveness of the proposed analytical method.

(2) The increase of one individual control period would lead to system instability.

(3) The enlargement of the disagreement between control periods of various DGs can also cause system instability.

(4) The asynchronization between control periods would give rise to steady-state deviation when adopting the conventional consensus control, whereas the deviation can 
be effectively eliminated using the improved ratio consensus algorithm proposed in this paper.

(5) The proposed algorithm would be effective regardless of load variation or topology switch.

\section{Conclusions}

In this paper, the stability and convergence accuracy of the distributed secondary control of DC microgrids in the case of asynchronous sampling have been investigated. The stability condition for the sampling periods of DGs is presented based on the periodicity of sampling time offset. Considering the convergence deviation caused by information asynchrony, an accurate distributed control algorithm based on ratio consensus is proposed, where an accurate solution can be achieved by real-time estimation and compensation of deviations. Through simulation testing, the following conclusions can be drawn:

- The system could come to be unstable when any individual control period becomes larger.

- Expansion of asynchronous degree can also lead to system instability.

- Steady-state deviation would occur in case of asynchronous control periods when adopting the conventional consensus control, whereas the deviation can be effectively eliminated using the proposed control method.

Author Contributions: Methodology, G.L.; software, Y.H.; writing-original draft preparation, Y.H.; writing-review and editing, S.L.; supervision, G.L. All authors have read and agreed to the published version of the manuscript.

Funding: This work was supported in part by the National Science Foundation of China under Grant 52007031, the National Science Foundation of Jiangsu Province under Grant BK20200404, the "Zhishan" Yong Scholars Programs of Southeast University and the Project Funding for Jiangsu Provincial Key Laboratory of Smart Grid Technology and Equipment, Southeast University.

Institutional Review Board Statement: Not applicable.

Informed Consent Statement: Not applicable.

Conflicts of Interest: The authors declare no conflict of interest.

\section{Appendix A}

Statement: $1 / p_{i j}$ is the probability that the time deviation is equal to $s_{i j, m}$ for any $m \in\left[1, p_{i j}\right]$.

Explanation: Assume $T=\operatorname{lcm}\left(T_{i}, T_{j}\right)=p_{i j} T_{i}$ and there exists a time deviation value $s$ that occurs more than once within period $T$.

According to the hypothesis, there must be integers $m_{1}, m_{2} \in\left[1, p_{i j}\right]$, which satisfy:

$$
\bmod \left(\frac{m_{1} T_{i}}{T_{j}}\right)=\bmod \left(\frac{m_{2} T_{i}}{T_{j}}\right)
$$

Thereby, there are positive integers $k_{1}, k_{2}$ meet the equation:

$$
m_{1} T_{i}-k_{1} T_{j}=m_{2} T_{i}-k_{2} T_{j}
$$

Rearranging the equation, it can be obtained that:

$$
\left(m_{1}-m_{2}\right) T_{i}=\left(k_{1}-k_{2}\right) T_{j}=T^{\prime}
$$

i.e., $T^{\prime}$ is the common multiple of $T_{i}$ and $T_{j}$. Meanwhile, given that $T$ is the least common multiple of $T_{i}$ and $T_{j}$, it can be inferred that $T^{\prime}=c T$, and thus $m_{1}-m_{2}=c p_{i j}$, where $c$ is a positive integer. Whereas the result that $m_{1}=m_{2}+c p_{i j}$ contradicts the assumption that $m_{1}$, $m_{2} \in\left[1, p_{i j}\right]$, implying the invalidation of the hypothesis. Therefore, each time deviation value can only occur once within period $T$. Further, consider the secondary control of the $i$ th and $j$ th DG is synchronous at $t_{0}$, they will synchronize again at $t_{0}+T$. Thus, it can be 
inferred that the change of the time deviation of information transmission between the $i$ th and $j$ th DG is periodic with respect to $T$.

Synthesize the above analysis, the conclusion can be drawn that it is equiprobable for the time deviation to be equal to any possible value $s_{i j, m}$ during a certain information transmission from the $j$ th DG to the $i$ th DG.

$$
\begin{aligned}
& A_{1}=\left[\begin{array}{c}
A_{11} \\
I_{n \cdot r_{\max } \times n \cdot r_{\max }}[0]_{n \cdot r_{\max } \times n \cdot h}
\end{array}\right]_{n \cdot\left(h+r_{\max }\right) \times n \cdot\left(h+r_{\max }\right)} \\
& \text { where } \quad A_{11} \quad\left[\begin{array}{c}
C^{\prime}{ }_{n \times n \cdot\left(h+r_{\max }\right)} \\
\vdots \\
C^{\prime}{ }_{n \times n} \cdot\left(h+r_{\max }\right)
\end{array}\right] \quad \text { and } \\
& C^{\prime} \quad\left[\begin{array}{lll}
{[0]_{n \times n \cdot(h-1)}} & H_{n \times n} & f_{n \times n \cdot r_{\max }}
\end{array}\right]_{n \times n \cdot\left(h+r_{\max }\right)} \text { with } \\
& H_{n \times n}=\left[\begin{array}{cccc}
1-\varepsilon T_{1} & \frac{\varepsilon T_{1}}{p_{12}} & \cdots & \frac{\varepsilon T_{1}}{p_{1 n}} \\
\frac{\varepsilon T_{2}}{p_{21}} & 1-\varepsilon T_{2} & \cdots & \frac{\varepsilon T_{2}}{p_{2 n}} \\
\vdots & \vdots & \ddots & \vdots \\
\frac{\varepsilon T_{n}}{p_{n 1}} & \frac{\varepsilon T_{n}}{p_{n 2}} & \cdots & 1-\varepsilon T_{n}
\end{array}\right]_{n \times n} . \\
& f(i, n \cdot(m-1)+j)=\left\{\begin{array}{cc}
\frac{\varepsilon T_{i}}{p_{i j}} & i f \exists k \in\left[1, p_{i j}\right], r_{i j, k}=m \\
0 & \text { otherwise }
\end{array}\right. \\
& R_{1}=\left[\begin{array}{ccc}
I_{n \times n} & {[0]_{n \times n}} & {[0]_{n \times n}} \\
M+k_{p 1} L M & I_{n \times n} & k_{p 2} \cdot I_{n \times n} \\
{[0]_{n \times n}} & -I_{n \times n} & I_{n \times n}
\end{array}\right] \\
& B^{\prime}=\left[\begin{array}{ccc}
S_{1 n \times n} & \omega_{f} T_{s}\left(I+V Y^{\prime}\right) & {[0]_{n \times n}} \\
-k_{i 1} L M & {[0]_{n \times n}} & -k_{i 2} \cdot I_{n \times n} \\
{[0]_{n \times n}} & {[0]_{n \times n}} & -k_{3} L
\end{array}\right] \\
& R_{2}=\left[\begin{array}{cc}
I_{n \times n} & {[0]_{n \times n}} \\
M_{n \times n} & I_{n \times n}
\end{array}\right] \\
& B_{1}=\left[\begin{array}{cc}
S_{2 n \times n} & \omega_{f} T_{S}\left(I+V Y^{\prime}\right) \\
M_{n \times n} & I_{n \times n}
\end{array}\right] \\
& C_{1}=\left[\begin{array}{cc}
{[0]_{n \times n}} & {[0]_{n \times n}} \\
{[0]_{n \times n}} & I_{n \times n}
\end{array}\right] \\
& I_{n \times n}=\left[\begin{array}{cccc}
1 & 0 & \cdots & 0 \\
0 & 1 & & \vdots \\
\vdots & & \ddots & 0 \\
0 & \cdots & 0 & 1
\end{array}\right]_{n \times n} \\
& S_{1 n \times n}=\left[\begin{array}{cccc}
-\omega_{f} & 0 & \cdots & 0 \\
0 & -\omega_{f} & & \vdots \\
\vdots & & \ddots & 0 \\
0 & \cdots & 0 & -\omega_{f}
\end{array}\right]_{n \times n}
\end{aligned}
$$




$$
\begin{aligned}
& S_{2 n \times n}=\left[\begin{array}{cccc}
1-\omega_{f} T_{s} & 0 & \cdots & 0 \\
0 & 1-\omega_{f} T_{s} & & \vdots \\
\vdots & & \ddots & 0 \\
0 & \cdots & 0 & 1-\omega_{f} T_{s}
\end{array}\right]_{n \times n} \\
& W_{i 2}=k_{p 1}\left[\begin{array}{ll}
M_{i 1} & {[0]_{1 \times n}}
\end{array}\right]_{1 \times 2 n^{\prime}}, W_{i 3}=-W_{i 2}+k_{i 1} T_{i}\left[\begin{array}{ll}
M_{i 1} & {[0]_{1 \times n}}
\end{array}\right]_{1 \times 2 n^{\prime}}, G_{i 1}=-k_{p 2} \cdot e_{i} \text {, } \\
& G_{i 2}=-G_{i 1}-k_{i 2} T_{i} \cdot e_{i}, \quad W_{i 4}=\left[[0]_{1 \times n} \quad e_{i}\right]_{1 \times 2 n^{\prime}}, G_{i 3}=e_{i}+k_{i 3} T_{i} L_{i 1} \text { with } \\
& e_{i} \quad=\quad\left[\begin{array}{lllllll}
0 & \cdots & 0 & \underbrace{1}_{i \text { th }} & 0 & \cdots & 0
\end{array}\right]_{1 \times n} \text {, } \\
& M_{i 1} \quad=\quad\left[\begin{array}{lllllll}
m_{1} & \cdots & m_{i-1} & -d_{i} m_{i} & m_{i+1} & \cdots & m_{n}
\end{array}\right]_{1 \times n} \quad \text { and } \\
& L_{i 1}=\left[\begin{array}{lllllll}
1 & \cdots & 1 & -d_{i} & 1 & \cdots & 1
\end{array}\right]_{1 \times n} .
\end{aligned}
$$

The acquisition of $D_{1}$ can be implemented as follows: for $l \in[1, n]$, suppose $n_{i}$ control instants are included in a secondary control period of the $i$ th DG, i.e., $n_{i}=T_{i} / T_{\text {s }}$; $D_{1}^{(0)}=I_{3 n \times 3 n}$ if $t_{k}^{l+1}$ is not a secondary control instant, i.e., $\bmod \left(l / n_{i}\right) \neq 0$ for any $i$

$$
D_{1}^{(l)}=\bar{W}_{1} D_{1}^{(l-1)}
$$

where $\bar{W}_{1}=\left[\begin{array}{cc}W_{1} & {[0]_{2 n \times n}} \\ {[0]_{n \times 2 n}} & I_{n \times n}\end{array}\right]_{3 n \times 3 n}$, if $t_{k}^{l+1}$ is a secondary control instant of the $i$ th DG, i.e., $\bmod \left(l / n_{i}\right)=0$.

$$
\begin{gathered}
D_{1}^{(l)}=\bar{R}^{-1}\left(\bar{W} D_{1}^{(l-1)}+\bar{H} V_{i}\right) \\
\bar{R}=\left[\begin{array}{cc}
I_{2 n \times 2 n}-H_{1} W_{2} & -H_{1} G_{1} \\
-W_{4} & I_{2 n \times 2 n}
\end{array}\right]_{3 n \times 3 n} \\
\bar{W}=\left[\begin{array}{cc}
W_{1} & {[0]_{2 n \times n}} \\
{[0]_{n \times 2 n}} & {[0]_{n \times n}}
\end{array}\right]_{3 n \times 3 n} \\
\bar{H}=\left[\begin{array}{cc}
H_{1} W_{3} & H_{1} G_{2} \\
-W_{4} & G_{3}
\end{array}\right]_{3 n \times 3 n} \text { with } W_{2}=e_{i}^{T} W_{i 2}, W_{3}=e_{i}^{T} W_{i 3}, G_{1}=e_{i}^{T} G_{i 1}, G_{2}=e_{i}^{T} G_{i 2}, \\
W_{4}=e_{i}^{T} W_{i 4}, G_{3}=e_{i}^{T} G_{i 3} . \\
\text { Then } V_{i} \text { will be updated as } V_{i}=D_{1}^{(l)} . \\
\text { After } n \text { iterations, it can be obtained that } D_{1}=D_{1}^{(n)} .
\end{gathered}
$$

\section{References}

1. Yu, K.; Ai, Q.; Wang, S.; Ni, J.; Lv, T. Analysis and optimization of droop controller for microgrid system based on small-signal dynamic model. IEEE Trans. Smart Grid 2016, 7, 695-705. [CrossRef]

2. Lou, G.; Gu, W.; Xu, Y.; Jin, W.; Du, X. Stability robustness for secondary voltage control in autonomous microgrids with consideration of communication delays. IEEE Trans. Power Syst. 2018, 33, 4164-4178. [CrossRef]

3. Liu, S.; Wang, X.; Liu, P.X. Impact of communication delays on secondary frequency control in an islanded microgrid. IEEE Trans. Ind. Electron. 2015, 62, 2021-2031. [CrossRef]

4. He, J.; Li, Y.W. Analysis, design, and implementation of virtual impedance for power electronics interfaced distributed generation. IEEE Trans. Ind. Appl. 2011, 47, 2525-2538. [CrossRef]

5. Li, Z.; Shahidehpour, M. Small-signal modeling and stability analysis of hybrid AC/DC microgrids. IEEE Trans. Smart Grid 2019, 10, 2080-2095. [CrossRef]

6. Dong, M.; Li, L.; Nie, Y.; Song, D.; Yang, J. Stability analysis of a novel distributed secondary control considering communication delay in DC microgrids. IEEE Trans. Smart Grid 2019, 10, 6690-6700. [CrossRef]

7. Dou, C.; Yue, D.; Guerrero, J.M.; Xie, X.; Hu, S. Multiagent system-based distributed coordinated control for radial DC microgrid considering transmission time delays. IEEE Trans. Smart Grid 2017, 8, 2370-2381. [CrossRef]

8. de Nadai Nascimento, B.; Zambroni de Souza, A.C.; Marujo, D.; Sarmiento, J.E.; Alvez, C.A.; Portelinha, F.M., Jr; de Carvalho Costa, J.G. Centralised secondary control for islanded microgrids. IET Renew. Power Gener. 2020, 14, 1502-1511. [CrossRef]

9. Qian, T.; Liu, Y.; Zhang, W.; Tang, W.; Shahidehpour, M. Event-triggered updating method in centralized and distributed secondary controls for islanded microgrid restoration. IEEE Trans. Smart Grid 2020, 11, 1387-1395. [CrossRef] 
10. Guo, F.; Xu, Q.; Wen, C.; Wang, L.; Wang, P. Distributed secondary control for power allocation and voltage restoration in islanded DC microgrids. IEEE Trans. Sustain. Energy 2018, 9, 1857-1869. [CrossRef]

11. Nasirian, V.; Moayedi, S.; Davoudi, A.; Lewis, F.L. Distributed cooperative control of DC microgrids. IEEE Trans. Power Electron. 2015, 30, 2288-2303. [CrossRef]

12. Xing, L.; Mishra, Y.; Guo, F.; Lin, P.; Yang, Y.; Ledwich, G.; Tian, Y. Distributed secondary control for current sharing and voltage restoration in DC microgrid. IEEE Trans. Smart Grid 2020, 11, 2487-2497. [CrossRef]

13. Baranwal, M.; Askarian, A.; Salapaka, S.; Salapaka, M. A distributed architecture for robust and optimal control of DC microgrids. IEEE Trans. Ind. Electron. 2019, 66, 3082-3092. [CrossRef]

14. Morstyn, T.; Hredzak, B.; Demetriades, G.D.; Agelidis, V.G. Unified distributed control for DC microgrid operating modes. IEEE Trans. Power Syst. 2016, 31, 802-812. [CrossRef]

15. Fan, B.; Peng, J.; Yang, Q.; Liu, W. Distributed periodic event-triggered algorithm for current sharing and voltage regulation in DC microgrids. IEEE Trans. Smart Grid 2020, 11, 577-589. [CrossRef]

16. Wang, P.; Lu, X.; Yang, X.; Wang, W.; Xu, D. An improved distributed secondary control method for DC microgrids with enhanced dynamic current sharing performance. IEEE Trans. Power Electron. 2016, 31, 6658-6673. [CrossRef]

17. Liu, X.; Jiang, H.; Wang, Y.; He, H. A distributed iterative learning framework for DC microgrids: Current sharing and voltage regulation. IEEE Trans. Emerg. Top. Comput. Intell. 2020, 4, 119-129. [CrossRef]

18. Wang, C.; Zhang, T.; Luo, F.; Li, F.; Liu, Y. Impacts of cyber system on microgrid operational reliability. IEEE Trans. Smart Grid 2019, 10, 105-115. [CrossRef]

19. Mattioni, M. On multiconsensus of multi-agent systems under aperiodic and asynchronous sampling. IEEE Contr. Syst. Lett. 2020, 4, 839-844. [CrossRef]

20. Zhan, J.; Li, X. Asynchronous consensus of multiple double- integrator agents with arbitrary sampling intervals and communication delays. IEEE Trans. Circuits Syst. I Reg. Pap. 2015, 62, 2301-2311. [CrossRef]

21. Lou, G.; Gu, W.; Lu, X.; Xu, Y.; Hong, H. Distributed secondary voltage control in islanded microgrids with consideration of communication network and time delays. IEEE Trans. Smart Grid 2020, 11, 3702-3715. [CrossRef]

22. Coelho, E.A.; Wu, D.; Guerrero, J.M.; Vasquez, J.C.; Dragicevic, T.; Stefanovic, C.; Popovski, P. Small-signal analysis of the microgrid secondary control considering a communication time delay. IEEE Trans. Ind. Electron. 2016, 63, 6257-6269. [CrossRef]

23. Savaghebi, M.; Jalilian, A.; Vasquez, J.C.; Guerrero, J.M. Secondary Control Scheme for Voltage Unbalance Compensation in an Islanded Droop-Controlled Microgrid. IEEE Trans. Smart Grid 2012, 3, 797-807. [CrossRef]

24. Guerrero, J.M.; Vasquez, J.C.; Matas, J.; de Vicuna, L.G.; Castilla, M. Hierarchical control of droop-controlled AC and DC microgrids-a general approach toward standardization. IEEE Trans. Ind. Electron. 2011, 58, 158-172. [CrossRef]

25. Lai, J.; Lu, X.; Yu, X.; Monti, A.; Zhou, H. Distributed voltage regulation for cyber-physical microgrids with coupling delays and slow switching topologies. IEEE Trans. Syst. Man Cybern. A Syst. 2020, 50, 100-110. [CrossRef]

26. Meng, X.; Liu, J.; Liu, Z. A Generalized Droop Control for Grid-Supporting Inverter Based on Comparison between Traditional Droop Control and Virtual Synchronous Generator Control. IEEE Trans. Power Electron. 2019, 34, 5416-5438. [CrossRef]

27. Zhong, Q. Robust Droop Controller for Accurate Proportional Load Sharing Among Inverters Operated in Parallel. IEEE Trans. Ind. Electron. 2013, 60, 1281-1290. [CrossRef]

28. Fatihcan, M.A. Consensus in networks under transmission delays and the normalized Laplacian. IFAC Proc. Vol. 2010, 43, 277-282.

29. Khosravi, A.; Kavian, Y.S. Broadcast gossip ratio consensus: Asynchronous distributed averaging in strongly connected networks. IEEE Trans. Signal Process. 2017, 65, 119-129. [CrossRef]

30. Du, Y.; Tu, H.; Yu, H.; Lukic, S. Accurate consensus-based distributed averaging with variable time delay in support of distributed secondary control algorithms. IEEE Trans. Smart Grid 2020, 11, 2918-2928. [CrossRef] 